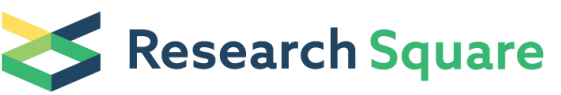 \\ Preprints are preliminary reports that have not undergone peer review. \\ They should not be considered conclusive, used to inform clinical practice, \\ or referenced by the media as validated information.
}

\section{Impact of Keratoconus Stage on Outcome After Corneal Crosslinking}

\section{Caroline Julia Gassel}

Centre for Ophthalmology, University Eye Hospital, Eberhard Karls University Tübingen

Daniel Röck

Centre for Ophthalmology, University Eye Hospital, Eberhard Karls University Tübingen

\section{Eva-Maria Konrad}

Centre for Ophthalmology, University Eye Hospital, Eberhard Karls University Tübingen

\section{Gunnar Blumenstock}

Institute for Clinical Epidemiology and Applied Biometry, Eberhard Karls University Tübingen

Karl Ulrich Bartz-Schmidt

Centre for Ophthalmology, University Eye Hospital, Eberhard Karls University Tübingen

Tobias Röck ( $\square$ tobias.roeck@med.uni-tuebingen.de)

Centre for Ophthalmology, University Eye Hospital, Eberhard Karls University Tübingen

\section{Research Article}

Keywords: Keratoconus, crosslinking, Krumeich, CXL, corneal crosslinking

Posted Date: November 8th, 2021

DOI: https://doi.org/10.21203/rs.3.rs-1049435/v1

License: @ (1) This work is licensed under a Creative Commons Attribution 4.0 International License. Read Full License 


\section{Abstract}

Background: This study aimed to analyze if the outcome after corneal crosslinking (CXL) in progressive keratoconus patients depends on the stage in which the procedure is performed. This knowledge would help to improve success of CXL and facilitate defining surgery indications in those patients.

Methods: In this retrospective study, 124 consecutive eyes of 100 patients with progressive keratoconus undergoing corneal CXL at the University Eye Hospital Tübingen were included. The eyes were graded according to modified Krumeich stages depending on induced myopia or astigmatism, corneal radii, minimum corneal thickness, and morphological changes. The observation period took place from November 2008 to September 2018. Preoperatively, 12 and 24 months after CXL, the best corrected visual acuity (BCVA) was determined and astigmatism as well as keratometric parameters (Kmax, Kmin, central corneal thickness (CCT), minimum corneal thickness (MCT)) were measured by means of a Scheimpflug camera system.

Results: BCVA results showed significant differences between the modified Krumeich stages 12 months $(p=0.014)$ and 24 months postoperatively ( $p=0.032)$. Also, astigmatism differed significantly among the stages 24 months after $C X L(p=0.023)$; however, after 12 months, no significant differences between the groups were detectable. In terms of Kmax, Kmin, CCT, and MCT, no significant differences between the Krumeich stages were observed.

Conclusions: BCVA showed a significantly higher improvement after CXL in the early stage of keratoconus compared to a higher stage. However, the postinterventional keratometric values did not differ significantly between the different modified Krumeich stages. The significantly higher improvement in BCVA after CXL in the early stage might indicate that earlier intervention provides a higher subjective benefit to the individual. Further studies with larger sample sizes are needed to confirm these findings.

\section{Background}

Keratoconus is a degenerative disease leading to a thinning and ectasia of the cornea with an onset typically in early adulthood (1). It usually occurs bilaterally but can present with asymmetric manifestation on both eyes. In most cases keratoconus occurs sporadically, but genetic factors may also play a role since an accumulation of cases in affected families with an apparently autosomal dominant pattern of inheritance is reported (2). A comorbidity with Down's syndrome, mitral valve prolapse and Leber congenital amaurosis is reported (3). Additionally, associations with atopic dermatitis, asthma, allergic rhinitis and diabetes mellitus were detected (4).

Patients with keratoconus suffer from reduced vision due to irregular astigmatism and myopia, often affecting the patients' quality of life (5). In advanced stages of the disease, corneal scarring can develop, further impairing vision (6). The characteristic Fleischer's ring, a thin pigmented circular line of hemosiderin depositions surrounding the cone base can occur (7). Another characteristic feature are fine vertical lines in the posterior stroma also termed Vogt's striae (8). Munson's sign, a protrusion of the lower eyelid during downgaze is another typical sign presented by keratoconus patients. In advanced findings, tears in the Descemet membrane lead to a leakage of aqueous humor into corneal stroma, resulting in an acute stromal oedema, also referred to as corneal hydrops (9).

Stages of keratoconus can be categorized on the basis of clinical characteristics using a classification Krumeich established almost a quarter of a century ago $(10,11)$. Krumeich differentiates four stages of keratoconus. Therapeutic management differs depending on the stage of keratoconus. In early stages, rigid gas permeable contact lenses are used to correct vision impairment (12). In more advanced stages with topographically established progression, corneal surgery or corneal crosslinking $(\mathrm{CXL})$ are required. $\mathrm{CXL}$, which was recognized as a statutory health insurance benefit in Germany by the Federal Joint Committee in mid-2018, is an established and safe treatment option for progressive keratoconus. Penetrating keratoplasty and deep anterior lamellar keratoplasty (DALK) are common methods but exhibit many potential complications (13). If stage IV of the Krumeich classification system is reached, corneal scars exist and CXL is no longer an appropriate treatment option. In these cases, penetrating keratoplasty or DALK are the methods of choice $(14,15)$.

Ultraviolet corneal CXL is a newer and less invasive procedure. In 1997, it was first demonstrated that the combination of riboflavin and ultraviolet (UV) irradiation is able to alter the biomechanical properties of the cornea (16). UV-A light and riboflavin are applied in order to induce a stiffening of the cornea. Riboflavin serves as a photosensitizer in this process. When exposed to UV-A light, riboflavin produces reactive oxygen species inducing covalent bonds between collagen molecules and proteoglycans (17). As the chemical cross-links between collagen fibrils are weaker than normal in corneal ectasias like keratoconus (18), the increase in rigidity induced by CXL helps to stabilize the cornea and halt further progression of the disease. In April 2016, CXL was approved for the treatment of progressive keratoconus by United States Food and Drug Administration (19). Meanwhile, CXL has become a standard treatment for stopping further progression of keratoconus (2). 
Overall, CXL has a good safety profile. Complications are rare in comparison to keratoplasty. They include corneal haze, scarring, postoperative infections or ulcers, endothelial damage, reactivation of herpes viridae infections and sterile corneal stromal infiltrates (20, 21).

In the beginning of the 21 st century, keratoconus was a common indication for penetrating keratoplasty. Our study group reported in 2018 that keratoconus was the most common surgical indication for corneal transplantation at the University Eye Hospital in Tübingen from 2004 to 2009. However, after introduction of CXL in 2008, the need of corneal transplants in keratoconus patients decreased significantly (22). Other studies reported similar changes in keratoplasty indications after implementation of CXL $(23,24)$.

CXL was shown to be effective in the improvement of visual acuity and keratometric parameters in the long term (25, 26). Nevertheless, there are no specific guidelines on the ideal point in time for CXL implementation. Therefore, this study was designed to investigate whether the success of CXL depends on the Krumeich stage of keratoconus in which the procedure is performed.

To the best of our knowledge, a study evaluating the influence of the stages of keratoconus on the results of CXL has never been undertaken before.

\section{Methods}

\section{Study realization}

In this retrospective study, 124 consecutive eyes of 100 patients were included between November 2008 and September 2018 . The inclusion criteria comprised progression of the keratoconus, primary CXL treatment and a preoperative follow-up period of at least 12 months.

Progression was defined as the following changes within one year: increase in maximum anterior sagittal curvature (Kmax) by $>1 \mathrm{dpt}$ and/or decrease in minimum corneal thickness by $\geq 5 \%$. Eyes with previous trauma or corneal procedures were excluded.

CXL was performed according to a slightly modified Dresden protocol as previously described (25).

The eyes were categorized based on modified Krumeich stages. We adjusted the Krumeich grading system and differentiated three different groups: group I, group II and group III. Our modified classification system is mainly based on Kmax. Group I includes the patients with Kmax $<48 \mathrm{dpt}$, group II with $\mathrm{Kmax}=48-53 \mathrm{dpt}$ and group III $>53 \mathrm{dpt}$, respectively. The defining parameters of the modified grades are presented in Table 1.

Table 1

- Definition of the groups investigated in this study.

\begin{tabular}{|lll|}
\hline Stage & Kmax & Morphological characteristics \\
\hline Group I & $<48 \mathrm{dpt}$ & Vogt's striae, no corneal scars, corneal thickness $\geq 400 \mu \mathrm{m}$ \\
\hline Group II & $48-53 \mathrm{dpt}$ & No central scars, corneal thickness $\geq 400 \mu \mathrm{m}$ \\
\hline Group III & $>53 \mathrm{dpt}$ & No central scars, corneal thickness $\geq 400 \mu \mathrm{m}$ \\
\hline
\end{tabular}

Modified Krumeich classification system of keratoconus stages defined by the presented parameters.

Best corrected visual acuity (BCVA) was determined in logMAR before and after CXL by means of refractometry with additional subjective comparison. The patients did not wear rigid contact lenses during visual acuity testing. A camera system based on Scheimpflug's principle (Orbscan II, Bausch\&Lomb, Rochester, NY, USA) was used to obtain the corneal keratometric data (Kmax, Kmin, astigmatism, minimum corneal thickness, central corneal thickness). The measurements took place preoperatively and 12 as well as 24 months postoperatively.

All methods have been performed in accordance with the Declaration of Helsinki. The study was approved by the Ethics Committee of the medical faculty of Eberhard Karls University of Tübingen and the University Hospital of Tübingen (740/2018B02).

\section{Statistical Analysis}

Quantitative measurement data are reported with the mean and standard deviation. Additionally, measured data is visualised using grouped bar charts with time of observation (preoperative, 12 and 24 months of follow-up, respectively) as the primary, and modified Krumeich stages as the secondary variable. To compare CXL outcome across modified Krumeich classes at 12 and at 24 months, respectively, analysis of covariance (ANCOVA) was performed with baseline values as covariate. The level of statistical significance was predefined at $a=0.05$. If applicable, pairwise post-hoc comparisons of effects were carried out using the Tukey-Kramer HSD test with adjustment for multiplicity. For all statistical analyses, the JMP® 14.2 statistical software was used (SAS Institute Inc., Cary, NC, USA). 


\section{Results}

\section{Patient population}

Regarding the gender distribution, 110 of the 124 study eyes were from males and 14 were from females. On average, the patients were $23.0 \pm$ 8.3 years old.

\section{Significant differences in visual acuity and astigmatism after crosslinking according to modified Krumeich stages}

The preoperative BCVA was $0.16 \pm 0.17$ logMAR in group I, $0.26 \pm 0.23$ logMAR in group II and $0.36 \pm 0.14$ in group III. 12 months following CXL, BCVA in group I was $0.1 \pm 0.12 \log M A R, 0.23 \pm 0.17 \log M A R$ in group II and $0.25 \pm 0.11 \log$ MAR in group III. After 24 months, BCVA was $0.1 \pm 0.14$ logMAR in group I, $0.26 \pm 0.24 \log M A R$ in group II and $0.26 \pm 0.12 \log M A R$ in grup III. In terms of BCVA, significant differences between groups in the 12 months postoperative (post-op) group ( $p=0.014$ ) as well as in the 24 months post-op group ( $p=0.032)$ were observed compared to the preoperative assessment. The patients in group II had a significantly lower increase in visual acuity than those in group I (group II vs. I: $\mathrm{p}=0.011$ at 12 months post-op; $\mathrm{p}=0.03$ at 24 months postop).

Patients of group I presented with a mean astigmatism of $3.04 \pm 1.7 \mathrm{dpt}$. In group II, the mean astigmatism was $4.43 \pm 2.08 \mathrm{dpt}$, in group III $6.67 \pm 3.48 \mathrm{dpt} .12$ months post-op, astigmatisms of $2.98 \pm 1.61 \mathrm{dpt}$ in group I, $4.02 \pm 2.07 \mathrm{dpt}$ in group II and $5.77 \pm 3.1 \mathrm{dpt}$ in group III were measured. After 24 months, the astigmatism was $3.08 \pm 1.38 \mathrm{dpt}$ in group I, 3.82 $\pm 2.01 \mathrm{dpt}$ and $5.69 \pm 2.69 \mathrm{dpt}$ in groups II and III, respectively. Regarding astigmatism, results of the 12 months post-op patients did not differ significantly among the three groups ( $p=0.39)$. However, after 24 months, significant differences between the three groups were detectable $(p=0.023)$. Especially group II was able to achieve a significant improvement regarding astigmatism compared to group I (group II vs. I: p=0.003 24 months post-op).

\section{No significant impact of the modified Krumeich stages on keratometric parameters}

Preoperatively, average Kmax in group I was $45.4 \pm 2$ dpt, and $50.3 \pm 1.5$ dpt and $55.2 \pm 1.5$ dpt in groups II and III, respectively. At 12 months following $\mathrm{CXL}$, mean Kmax was measured $45.3 \pm 2.1 \mathrm{dpt}$ in group I, $49.2 \pm 1.9 \mathrm{dpt}$ in group II and $53.8 \pm 2.1$ in group III. At 24 months postintervention, the average Kmax was measured $45.2 \pm 2.2 \mathrm{dpt}$ in group I, $48.2 \pm 3.5 \mathrm{dpt}$ in group II and $53.9 \pm 1.7 \mathrm{dpt}$ in group III.

Measured Kmax values did not differ significantly between the three groups 12 months $(p=0.08)$ or 24 months ( $p=0.27)$ after surgery.

Regarding Kmin, preoperative average values were $42.4 \pm 1.7 \mathrm{dpt}$ in group I, $45.7 \pm 1.8 \mathrm{dpt}$ in group II and $48.6 \pm 3.3 \mathrm{dpt}$ in group III. One year after the procedure, $\mathrm{Kmin}$ was $42.3 \pm 1.8 \mathrm{dpt}$ in group I, $45 \pm 2.3 \mathrm{dpt}$ in group II and $48 \pm 2.7 \mathrm{dpt}$ in group III. 2 years following CXL, Kmin was $42.3 \pm 2$ dpt, $44.5 \pm 3.7 \mathrm{dpt}$ and $48.3 \pm 2.6 \mathrm{dpt}$ in the groups I, II and III.

The postoperative Kmin results did not show significant alterations among the modified stages either (12 months post-op: $\mathrm{p}=0.47$; 24 months post-op: $p=0.65)$.

Before CXL, mean MCT was $483.5 \pm 30.7 \mu \mathrm{m}$ in group I. Groups II and III presented with a mean MCT of $468.2 \pm 31.1 \mu \mathrm{m}$ and $450.5 \pm 36.5 \mu \mathrm{m}$ before the procedure. 12 months after $\mathrm{CXL}$, mean MCT was measured lower at $472.8 \pm 38.7 \mu \mathrm{m}, 472.8 \pm 38.7 \mu \mathrm{m}$ and $432 \pm 41.4 \mu \mathrm{m}$ in each group. After 2 years, the average MCT was $477.9 \pm 43.7 \mu \mathrm{m}, 446.2 \pm 52.1 \mu \mathrm{m}$ and $429.4 \pm 47 \mu \mathrm{m}$ in groups I-III.

No significant differences in minimum corneal thickness was noticed after $12(p=0.32)$ or 24 months ( $p=0.39)$ depending on the groups.

Before CXL, mean CCT was $511.3 \pm 30.8 \mu \mathrm{m}$ in group I, $491.5 \pm 25.7 \mu \mathrm{m}$ in group II and $479.9 \pm 35.6 \mu \mathrm{m}$ in group III. 12 months after the intervention, CCT values were lower at $500.6 \pm 34.7 \mathrm{~mm}$ in group I, $470.9 \pm 43.8 \mu \mathrm{m}$ in group II and $463.8 \pm 42.3 \mu \mathrm{m}$ in group III. After $24 \mathrm{months}$, $501.9 \pm 39.4 \mu \mathrm{m}$ in group I, $476.6 \pm 42.9 \mu \mathrm{m}$ in group II and $461.4 \pm 36 \mu \mathrm{m}$ in group III were measured.

Similar to the previous results, the central corneal thickness did not differ significantly between the different Krumeich stages 12 months $(p=0.24)$ or 24 months $(p=0.60)$ post-op.

In Figure 1 and Table 2, the respective BCVA and keratometric values at the respective measurement times in the three groups are presented.

Data presented as mean \pm SD preoperatively and 12 and 24 months following corneal CXL. a) BCVA, b) astigmatism, c) Kmax, d) Kmin, e) $M C T, f)$ CCT. Statistically significant group differences ( $\left.{ }^{*} p<0.05\right)$ were observed for BCVA (12 months and 24 months post-op) and astigmatism (24 months post-op) 
Table 2

- BCVA, astigmatism and keratometric values of the three groups preoperatively and after 12 and 24 months.

\begin{tabular}{|llllllllcc|}
\hline \multicolumn{3}{|c}{ preop } & & \multicolumn{3}{c|}{12 months } & & \multicolumn{2}{c}{24 months } \\
\hline Group & I & II & III & I & II & III & I & II \\
\hline BCVA & $0.16 \pm 0.17$ & $0.26 \pm 0.23$ & $0.36 \pm 0.14$ & $0.1 \pm 0.12$ & $0.23 \pm 0.17$ & $0.25 \pm 0.11$ & $0.1 \pm 0.14$ & $0.26 \pm 0.24$ & $0.26 \pm 0.12$ \\
\hline Astigm & $3.04 \pm 1.7$ & $4.43 \pm 2.08$ & $6.67 \pm 3.48$ & $2.98 \pm 1.61$ & $4.02 \pm 2.07$ & $5.77 \pm 3.1$ & $3.08 \pm 1.38$ & $3.82 \pm 2.01$ & $5.69 \pm 2.69$ \\
\hline Kmax & $45.4 \pm 2$ & $50.3 \pm 1.5$ & $55.2 \pm 1.5$ & $45.3 \pm 2.1$ & $49.2 \pm 1.9$ & $53.8 \pm 2.1$ & $45.2 \pm 2.2$ & $48.2 \pm 3.5$ & $53.9 \pm 1.7$ \\
\hline Kmin & $42.4 \pm 1.7$ & $45.7 \pm 1.8$ & $48.6 \pm 3.3$ & $42.3 \pm 1.8$ & $45 \pm 2.3$ & $48 \pm 2.7$ & $42.3 \pm 2$ & $44.5 \pm 3.7$ & $48.3 \pm 2.6$ \\
\hline MCT & $483.5 \pm 30.7$ & $468.2 \pm 31.1$ & $450.5 \pm 36.5$ & $472.8 \pm 38.7$ & $446.8 \pm 48.6$ & $432 \pm 41.4$ & $477.9 \pm 43.7$ & $446.2 \pm 52.1$ & $429.4 \pm 47$ \\
\hline CCT & $511.3 \pm 30.8$ & $491.5 \pm 25.7$ & $479.9 \pm 35.6$ & $500.6 \pm 34.7$ & $470.9 \pm 43.8$ & $463.8 \pm 42.3$ & $501.9 \pm 39.4$ & $476.6 \pm 42.9$ & $461.4 \pm 36$ \\
\hline
\end{tabular}

The means and standard deviations of the respective measurement data at the respective times in the different groups are presented. BCVA = best corrected visual acuity, Astigm = astigmatism, $\mathrm{Kmax}=$ maximum corneal curvature, $\mathrm{Kmin}=$ minimum corneal curvature, minimum corneal thickness, $\mathrm{MCT}=$ minimum corneal thickness, $\mathrm{CCT}=$ central corneal thickness

\section{Discussion}

Previous studies of our working group demonstrated a significant reduction of keratometric values and a significant increase in BCVA in a long-term follow-up after corneal CXL $(25,26)$. Usually, CXL is performed if the keratoconus progression parameters worsen within one year. These parameters include the reduction of the minimum corneal thickness by at least $5 \%$ or an increase of Kmax by one dioptre (27). However, there is no consensus about the best point in time to indicate the procedure. This raises the question if the pathomorphological stage, which can be defined by the Krumeich classification, has an influence on the postinterventional outcome. In the clinical routine, it is common for patients to ask whether it is better to have CXL performed sooner or later. So far, science has not been able to provide an answer to this important question. But this knowledge could help to determine the best time to indicate corneal CXL. By now, there is no literature about the best outcome of CXL depending on the Krumeich stages. This study is the first to investigate this relationship.

Interestingly, this study did not observe any significant differences between the modified Krumeich stages in terms of keratometric values. Nevertheless, BCVA and astigmatism differed significantly depending on the modified Krumeich stages. The patients in group I had a significantly higher improvement in BCVA than those in group II. This might show that patients subjectively benefit more from earlier intervention.

It is conceivable that there is a relationship between the influence of astigmatism and BCVA after CXL. However, it is unclear why the keratoconus stages have no significant impact on the improvement of keratometric parameters following the procedure. This would mean that CXL seems to be similarly effective in terms of keratometric parameters in each of the three modified Krumeich stages which we investigated in this study. Nevertheless, some points should be considered before drawing hasty conclusions.

The limitations of this study comprise the retrospective design with a possible selection bias and the impossibility to determine causation. Retrospective analyses are subject to confounding, which could potentially bias the results. Nonetheless, our data are supported by a relatively large case number of 124 eyes. To further investigate the impact of the keratoconus stage on post-CXL outcome, a large controlled prospective study should be performed. Another limitation that should be considered is the postoperative follow-up time of 24 months which might be too short to detect the true significant effects in the three different groups of this study. Recently, Vinciguerra et al. reported a study with a followup time after CXL up to 13 years (28). Tasci et al. retrospectively analysed long-term visual acuity, topographic and aberrometry results in a period up to 5 years after CXL. They concluded that CXL improves visual acuity and quality of vision by reducing higher-order aberrations and spherical aberrations, and stops the progression of keratoconus (29). However, they did not investigate which factors had a particularly positive or negative impact on the postinterventional results.

We plan to observe our patients 10 years after CXL in the future to gain further knowledge.

It may also be of interest to examine other factors which could influence the postinterventional results and therefore might play a role in the indication for CXL. For example, the patients' age or the actual rate of keratoconus progression may have an impact on the outcome. In a prospective comparative case series from Egypt, 22 keratoconus patients younger than 18 years underwent CXL and showed a significant improvement of visual acuity and keratometric values postoperatively, with no evidence of progression of keratoconus over 12 months (30). A 
retrospective study of 96 eyes from Turkey detected that patients older than 30 years, patients with a worse baseline BCVA and those with a thinner baseline pachymetry benefit most likely from CXL (31).

A validation study from Godefrooij et al. demonstrated that a lower visual acuity before CXL is the sole independent factor which can predict an improvement in visual acuity 12 months after the procedure. This finding suggests that patients with a lower vision are more likely to benefit from CXL (32). These data imply that in terms of visual acuity, higher stages of keratoconus benefit more from CXL. Since in our study we mainly used Kmax for classification into the different groups I, II and III, this does not contradict our results. A retrospective analysis from France postulates lower preoperative visual acuity, high astigmatism and advanced keratoconus as predictive factors for visual improvement after CXL (33).

In contrast to this, a retrospective analysis from Lebanon with 156 eyes and a minimum follow-up of 3 years demonstrated that a better preoperative visual acuity was associated with a higher vision improvement. A higher baseline Kmax was associated with a worse postinterventional visual acuity (34).

Overall, the literature contains very controversial results. In future, controlled prospective studies with a larger sample size should investigate the respective influence of the individual factors such as keratoconus stage, age and visual acuity. These findings could improve patient management and help define the optimal time to indicate CXL.

\section{Conclusion}

Our data suggest that patients benefit from corneal CXL regardless of modified Krumeich stages. However, we observed trends towards a comparatively higher improvement in visual acuity in the group with the early keratoconus stage. Larger controlled studies over longer observation periods should be conducted to verify these observations. These findings may optimize the management and indication of corneal CXL in keratoconus patients.

\section{Abbreviations}

BCVA

CCT

CXL

DALK

$\mathrm{Kmax}$

Kmin

MCT

UV best corrected visual acuity

central corneal thickness

corneal crosslinking

deep anterior lamellar keratoplasty

maximum corneal curvature

minimum corneal curvature

minimum corneal thickness

ultraviolet

\section{Declarations}

Ethics approval: All procedureshave been performed in accordance with the Declaration of Helsinki. This study was approved by a positive vote of the Ethics Committee of the Medical Faculty of Eberhard Karls University and the University Clinics Tübingen (740/2018BO2).

Consent for publication: As this study has a retrospective design with a high number of cases, it was not necessary to obtain a consent form. The procedure was approved by the Ethics Committee of the Medical Faculty of Eberhard Karls University and the University Clinics Tübingen.

Availability of data and materials: The datasets used and/or analysed during the current study are available from the corresponding author upon reasonable request.

Competing Interests: The authors declare that they have no competing interests.

Funding: None.

Author's Contribution: CG contributed to analysis and interpretation of data and drafted the manuscript. DR was involved in drafting and reviewing this manuscript. EMK contributed to data analysis. KUBS has given final approval. TR designed the study and contributed to data 
analysis and interpretation. All authors have read and approved the manuscript.

Acknowledgements: Not applicable.

\section{References}

1. Davidson AE, Hayes S, Hardcastle AJ, Tuft SJ. The pathogenesis of keratoconus. Eye (Lond). 2014;28(2):189-95.

2. Nielsen K, Hjortdal J, Pihlmann M, Corydon TJ. Update on the keratoconus genetics. Acta Ophthalmol. 2013;91(2):106-13.

3. Rabinowitz YS. Keratoconus. Surv Ophthalmol. 1998;42(4):297-319.

4. Moon JY, Lee J, Park YH, Park EC, Lee SH. Incidence of Keratoconus and Its Association with Systemic Comorbid Conditions: A Nationwide Cohort Study from South Korea. J Ophthalmol. 2020;2020:3493614.

5. Duncan JK, Belin MW, Borgstrom M. Assessing progression of keratoconus: novel tomographic determinants. Eye and vision (London, England). 2016;3:6.

6. Fournie P, Touboul D, Arne JL, Colin J, Malecaze F. [Keratoconus]. J Fr Ophtalmol. 2013;36(7):618-26.

7. Romero-Jimenez M, Santodomingo-Rubido J, Wolffsohn JS. Keratoconus: a review. Cont Lens Anterior Eye. 2010;33(4):157-66; quiz 205.

8. Grieve K, Ghoubay D, Georgeon C, Latour G, Nahas A, Plamann K, et al. Stromal striae: a new insight into corneal physiology and mechanics. Sci Rep. 2017;7(1):13584.

9. Maharana PK, Sharma N, Vajpayee RB. Acute corneal hydrops in keratoconus. Indian J Ophthalmol. 2013;61(8):461-4.

10. Krumeich JH, Daniel J, Knulle A. Live-epikeratophakia for keratoconus. J Cataract Refract Surg. 1998;24(4):456-63.

11. Krumeich JH, Daniel J. [Live epikeratophakia and deep lamellar keratoplasty for I-III stage-specific surgical treatment of keratoconus]. Klin Monbl Augenheilkd. 1997;211(2):94-100.

12. Downie LE, Lindsay RG. Contact lens management of keratoconus. Clin Exp Optom. 2015;98(4):299-311.

13. Parker JS, van Dijk K, Melles GR. Treatment options for advanced keratoconus: A review. Surv Ophthalmol. 2015;60(5):459-80.

14. Andreanos KD, Hashemi K, Petrelli M, Droutsas K, Georgalas I, Kymionis GD. Keratoconus Treatment Algorithm. Ophthalmology and Therapy. 2017;6(2):245-62.

15. Jones MN, Armitage WJ, Ayliffe W, Larkin DF, Kaye SB. Penetrating and deep anterior lamellar keratoplasty for keratoconus: a comparison of graft outcomes in the United kingdom. Invest Ophthalmol Vis Sci. 2009;50(12):5625-9.

16. Spörl E, Huhle M, Kasper M, Seiler T. [Increased rigidity of the cornea caused by intrastromal cross-linking]. Ophthalmologe. 1997;94(12):902-6.

17. Subasinghe SK, Ogbuehi KC, Dias GJ. Current perspectives on corneal collagen crosslinking (CXL). Graefes Arch Clin Exp Ophthalmol. 2018;256(8):1363-84.

18. Müller PL, Loeffler KU, Messmer E, Holz FG, Perdikakis G, Kohlhaas M, et al. Histological Corneal Alterations in Keratoconus After Crosslinking-Expansion of Findings. Cornea. 2020;39(3):333-41.

19. Evangelista CB, Hatch KM. Corneal Collagen Cross-Linking Complications. Seminars in ophthalmology. 2018;33(1):29-35.

20. Dhawan S, Rao K, Natrajan S. Complications of Corneal Collagen Cross-Linking. Journal of Ophthalmology. 2011;2011:869015.

21. Lang SJ, Reinhard T. Crosslinking bei Keratokonus. Klin Monbl Augenheilkd. 2016;233(06):713-6.

22. Röck T, Bartz-Schmidt KU, Röck D. Trends in corneal transplantation at the University Eye Hospital in Tübingen, Germany over the last 12 years: 2004 - 2015. PLoS One. 2018;13(6):e0198793.

23. Lang SJ, Bischoff M, Böhringer D, Seitz B, Reinhard T. Analysis of the changes in keratoplasty indications and preferred techniques. PLoS One. 2014;9(11):e112696.

24. Godefrooij DA, Gans R, Imhof SM, Wisse RP. Nationwide reduction in the number of corneal transplantations for keratoconus following the implementation of cross-linking. Acta Ophthalmol. 2016;94(7):675-8.

25. Konrad EM, Rock D, Blumenstock G, Bartz-Schmidt KU, Rock T. [Long-term experiences with corneal crosslinking in patients with progressive keratoconus at the University Eye Hospital in Tubingen, Germany]. Ophthalmologe. 2020;117(6):538-45.

26. Röck T KE, Röck D, Bramkamp M, Blumenstock G, Bartz-Schmidt KU. Successful Regression in Patients with Progressive Keratoconus by Corneal Crosslinking. Klin Monatsbl Augenheilkd. 2021;accepted but unpublished yet.

27. Maier P, Reinhard T. [Riboflavin UVA cross-linking for keratoconus]. Ophthalmologe. 2013;110(9):818-22.

28. Vinciguerra R, Pagano L, Borgia A, Montericcio A, Legrottaglie EF, Piscopo R, et al. Corneal Cross-linking for Progressive Keratoconus: Up to 13 Years of Follow-up. Journal of refractive surgery (Thorofare, NJ: 1995). 2020;36(12):838-43. 
29. Taşçı YY, Taşlıpınar G, Eyidoğan D, Saraç Ö, Çağıl N. Five-Year Long-Term Results of Standard Collagen Cross-Linking Therapy in Patients with Keratoconus. Turkish journal of ophthalmology. 2020;50(4):200-5.

30. Salman AG. Transepithelial corneal collagen crosslinking for progressive keratoconus in a pediatric age group. Journal of Cataract \& Refractive Surgery. 2013;39(8):1164-70.

31. Toprak I, Yaylalı V, Yildirim C. Factors affecting outcomes of corneal collagen crosslinking treatment. Eye. 2014;28(1):41-6.

32. Godefrooij DA, Boom K, Soeters N, Imhof SM, Wisse RP. Predictors for treatment outcomes after corneal crosslinking for keratoconus: a validation study. International ophthalmology. 2017;37(2):341-8.

33. De Angelis F, Rateau J, Destrieux C, Patat F, Pisella PJ. [Predictive factors for visual outcome after corneal collagen crosslinking treatment in progressive keratoconus: One-year refractive and topographic results]. Journal francais d'ophtalmologie. 2015;38(7):595-606.

34. Farhat R, Ghannam MK, Azar G, Nehme J, Sahyoun M, Hanna NG, et al. Safety, Efficacy, and Predictive Factors of Conventional Epithelium-Off Corneal Crosslinking in the Treatment of Progressive Keratoconus. J Ophthalmol. 2020;2020:7487186.

\section{Figures}

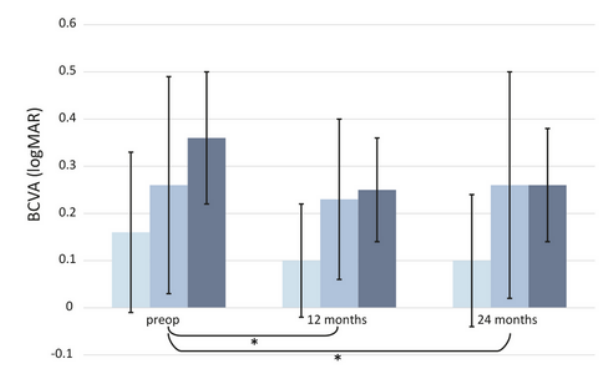

a
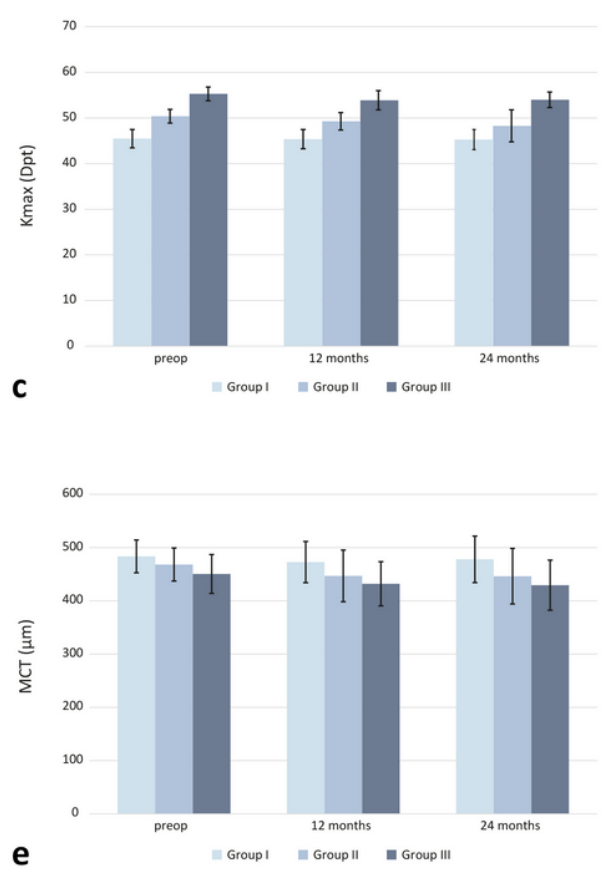
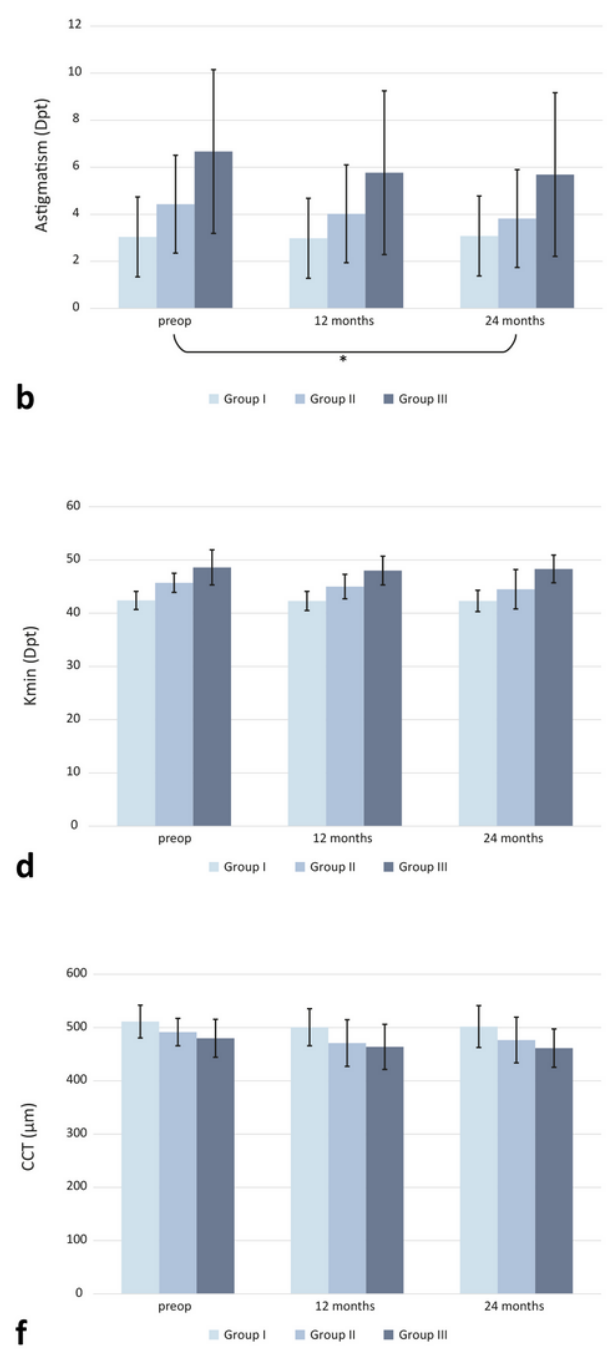

\section{Figure 1}

BCVA and keratometric parameters depending on the three groups Data presented as mean \pm SD preoperatively and 12 and 24 months following corneal CXL. a) BCVA, b) astigmatism, c) Kmax, d) Kmin, e) MCT, f) CCT. Statistically significant group differences (*p < 0.05) were observed for BCVA (12 months and 24 months post-op) and astigmatism (24 months post-op) 